\title{
Evaluación del springback mediante ensayos de doblado bajo tensión en condiciones de multiaxialidad típicas de los procesos de embutición profunda. Aplicación a chapa de acero inoxidable AISI $304 D_{D Q}{ }^{(\bullet)}$
}

\author{
V. Miguel*y**, J. Coello*y**, A. Martínez** y A. Calatayud*y**
}

\begin{abstract}
Resumen
En este trabajo se presenta una metodología para evaluar la recuperación elástica o springback de chapa de acero inoxidable AISI 304 DDQ basada en la realización de ensayos bajo tensión. A diferencia de los estudios existentes en la literatura, los ensayos realizados son efectuados en condiciones multiaxiales típicas de los procesos de embutición de chapa. Esto afecta fundamentalmente a las tensiones involucradas en el proceso así como al estado de endurecimiento que experimenta el material. La evaluación del springback se ha efectuado en dos áreas diferentes. En la zona de doblado se ha evaluado a partir del factor de recuperación definido como la razón entre el radio de doblado y el radio con el que queda finalmente el material. La zona de doblado y desdoblado se ha evaluado en base a la inversa del radio de curvatura de la chapa en dicha zona y teniendo en cuenta una longitud de arco en función de la relación de deformación practicada en el material. Los resultados encontrados permiten establecer que dentro del rango de valores de ángulo y de radio de doblado experimentados, el springback es independiente de la relación de embutición o de deformación practicada en el material. El radio de doblado tiene poca influencia en el rango de radios ensayados, siendo el ángulo de doblado la variable más significativa. Los resultados obtenidos son contrastados con los medidos en copas embutidas, encontrando un alto grado de concordancia.
\end{abstract}

\section{Springback evaluation by bending under tension tests in conditions of multiaxial stresses corresponding to deep drawing processes. Application to AISI 304 DDQ stainless steel sheet}

\begin{abstract}
In this paper, a methodology has been developed for evaluating the springback of AISI 304 DDQ stainless steel sheet based on a bending under tension test. The main difference of the methodology herein carried out is that tests are made under the multiaxial stresses state that take place in deep drawing processes. This affects to the level of stress value in the test and to the hardening state of the sheet. Springback evaluation has been done in two different areas. Bending area has been evaluated from elastic recovery ratio defined as the ratio between the bending radius after and before bending. Bending and unbending extreme has been studied from the measured curvature radius in this area and taking into account the geometric equivalence of the test with the drawing cups process. Results found allow to state that drawing ratio or deformation ratio have a negligible influence on the springback into the range of values experimented here. Bending radius has hardly influence as well while bending angle is the most significant variable. The results obtained are compared to those measured in deep-drawn cups, finding a great agreement.
\end{abstract}

Keywords

Springback; Bending under tension; AISI 304; Deep-drawing; Multiaxial.

\section{INTRODUCCIÓN}

Una de las dificultades que presentan los procesos de conformado de chapa es la recuperación elástica o springback del material tras el proceso. Este fenó- meno, en numerosas ocasiones da lugar a errores de imprecisión en las dimensiones de los productos finales, por lo que es muy importante conocer su magnitud, para poder considerarlo e integrarlo en el diseño de matrices de conformado. Muestra de la importan-

(•) Trabajo recibido el día 4 de septiembre de 2012 y aceptado en su forma final el día 3 de enero de 2013

* Escuela de Ingenieros Industriales de Albacete; Universidad de Castilla-La Mancha; Avda. España s/n 02006 Albacete.

** Instituto de Desarrollo Regional; Universidad de Castilla-La Mancha; Avda. España s/n 02006 Albacete. 
cia del fenómeno en el diseño de matrices son los trabajos existentes en la literatura dirigidos a corregir el fenómeno mediante el ajuste iterativo entre el resultado del efecto y las dimensiones y forma de la matriz ${ }^{[1 \text { y } 2]}$.

Tradicionalmente, la determinación del springback en los procesos específicos de doblado es la más tratada en la literatura, estableciéndose predicciones mediante tablas, gráficos y expresiones analíticas simplificadas, que permiten obtener de forma aproximada los valores de recuperación a partir de las propiedades de los materiales. Una de estas expresiones es la ecuación desarrollada por Gardiner ${ }^{[3]}$ que determina la recuperación elástica considerando las propiedades que definen el comportamiento elástico de los materiales utilizados. Marciniak ${ }^{[4]}$ proporciona una ecuación simplificada considerando la hipótesis de comportamiento elástico-plástico del material tras el proceso de doblado. Generalmente las soluciones que suelen aportar están referidas a materiales muy concretos y a ángulos de doblado de $90^{\circ}$. Los resultados que arrojan estas ecuaciones difieren en gran medida del springback obtenido en el conformado del material para otros materiales y condiciones de doblado ${ }^{[5]}$. Ello demuestra la necesidad de experimentar específicamente con los materiales que van a ser procesados considerando diferentes condiciones de conformado. Tekiner ${ }^{[6]}$ determina experimentalmente el springback de seis materiales diferentes sometidos a procesos de doblado en "U" y en "V", considerando como variables el espesor de la chapa y el ángulo de doblado $\left(15^{\circ}-90^{\circ}\right)$.

Son diversos los trabajos que desarrollan modelos analíticos, y métodos de simulación por elementos finitos para la determinación del springback en procesos de doblado simple ${ }^{[7}$ y 8$]$. Estos métodos permiten considerar muchos de los factores que intervienen en los procesos de deformación de chapa, como la determinación de la forma óptima del producto, la geometría inicial óptima de partida, la predicción del grosor de la chapa final y la predicción de la fractura. En general, se establece que el springback aumenta con la anisotropía del material y disminuye con el espesor de la chapa ${ }^{[7]}$. Leu ${ }^{[8]}$ formula un modelo matemático que estudia el efecto de la anisotropía de la chapa, $\mathrm{R}$, y el coeficiente de endurecimiento, $n$, sobre el springback en procesos de doblado simple, comparando los resultados analíticos obtenidos con datos experimentales. Los resultados demuestran que el springback es directamente proporcional al valor de $\mathrm{R}$ e inversamente proporcional al coeficiente de endurecimiento, $n$, y a la relación $t / 2 r$ entre el espesor, $t$, y el radio de doblado, $r$.

Tekaslan et al..$^{[9]}$ a partir de datos experimentales correlaciona el springback con el ángulo de doblado en procesos de doblado en "V" realizados sobre chapa de acero inoxidable de 0,5; 0,75 y $1 \mathrm{~mm}$ de espesor. Para cada uno de los espesores obtienen un ajuste polinómico de segundo grado con un elevado índice de correlación. Un estudio similar es desarrollado por García-Romeu et al. ${ }^{[10]}$ en el que se llevan a cabo ensayos de doblado en "V", variando el ángulo de doblado entre $22^{\circ}$ y $90^{\circ}$. En este caso, los materiales evaluados son chapa de acero inoxidable y de aluminio con un espesor comprendido entre 1 y $3 \mathrm{~mm}$. Los resultados obtenidos por estos autores indican que los valores del springback para el acero inoxidable son muy superiores a los obtenidos por Tekaslan, aunque las tendencias de comportamiento son las mismas.

En condiciones de embutición de chapa, los procesos de doblado son efectuados bajo condiciones de multiaxialidad que difieren de las de los procesos de doblado simple. La descomposición de acciones típica de estos procesos se establece a partir de doblado bajo tensión del material endurecido por cortante puro ${ }^{[11]}$. La influencia de la anisotropía y otros factores como la fuerza del pisador y las condiciones de lubricación sobre el springback son estudiadas por Ragai et al. ${ }^{[12]}$. Estos autores determinan mediante simulación por elementos finitos y experimentalmente que el ángulo de springback disminuye con el incremento de la fuerza del pisador en procesos de embutición de chapa de acero inoxidable AISI 410. También comprueban que el lubricante produce un efecto similar a la disminución de la fuerza del pisador sobre el springback, ya que disminuye la fricción existente. Pourbograt et al. ${ }^{[13]}$ desarrollan un método para predecir el springback en procesos de embutición con deformación plana, verificándolo con datos obtenidos en ensayos de embutición-doblado de chapa de aluminio. Ling et al. ${ }^{[14]}$ determinan el springback mediante elementos finitos en procesos de doblado y embutición de copas cuadradas de acero inoxidable. Comparan los datos obtenidos con datos experimentales, observando que las diferencias entre ambos valores aumentan con la altura de las copas. Diversos autores ${ }^{[15}$ y 16$]$ justifican las diferencias encontradas entre los datos experimentales y los obtenidos mediante MEF, por las condiciones de contacto uniforme consideradas en estos modelos. En los procesos de conformado, el contacto entre las superficies de trabajo no es totalmente uniforme y por tanto son muy frecuentes las dudas sobre los valores de los parámetros de fricción necesarios en la simulación. Este problema, encontrado también por diversos autores ${ }^{[9,10}$ y 14-16], unido a la dificultad que existe en numerosas ocasiones de enfrentarse a una descripción correcta del mecanismo de deformación del material y a la complejidad que presentan estos métodos, constituyen las principales limitaciones de los procesos de simulación aplicados a la determinación del springback. Estos métodos se utilizan como 
una primera aproximación para el estudio del springback, siendo necesario, en los casos en los que se requiere una gran precisión, recurrir a la determinación experimental.

Matlock et al. ${ }^{[17-20]}$ realizan numerosos trabajos experimentales en los que determinan el springback de ensayos de doblado bajo tensión, estudiando la influencia de numerosos factores sobre la magnitud de este fenómeno: tensión a tracción, anchura de las muestras dobladas y relación $\mathrm{r} / \mathrm{t}$. En la zona de doblado consideran la longitud de arco en contacto con el cilindro, mientras que en la zona de doblado y estirado toman como longitud de arco la longitud de estirado, constante para todos los ensayos. Estos autores realizan un estudio muy completo del springback sobre el acero inoxidable. Sin embargo, podría considerarse que sus trabajos presentan dos limitaciones importantes. Una de ellas es la variación del ángulo de doblado, ya que todos los ensayos de doblado son a $90^{\circ}$ y otra es la fuerza a tracción considerada. Del mismo modo, otros autores que realizan ensayos de doblado bajo tensión ${ }^{[21-23]}$, consideran valores de fuerza próximos al límite elástico, por lo que la deformación practicada es muy inferior a la que tiene lugar

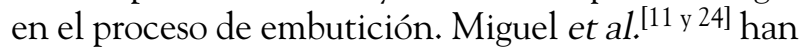
desarrollado y validado un ensayo de doblado bajo tensión sobre acero DC-05 y acero inoxidable AISI 304, en el que el material es sometido simultáneamente a doblado y deformación en condiciones de tracción-compresión biaxial demostrando que el estado previo del material influye significativamente sobre el comportamiento tribológico del mismo en el proceso de doblado.
En el presente trabajo se desarrolla una metodología para la determinación experimental del springback en ensayos de doblado y deformación en condiciones de tracción-compresión biaxial, típicas de las condiciones existentes en el radio de entrada a la matriz de embutición para el acero inoxidable AISI 304 DDQ, determinando la influencia de la presión, ángulo de doblado y radio de doblado en el proceso. Los resultados obtenidos reproducen bien las tendencias de comportamiento en copas embutidas y permiten evaluar el grado de influencia de las diferentes variables involucradas en el proceso.

\section{DESARROLLO EXPERIMENTAL}

\subsection{Materiales utilizados y variables estudiadas}

El material investigado en este trabajo es chapa de acero inoxidable austenítico AISI 304 DDQ de espesor, $\mathrm{t}, 0,8 \mathrm{~mm}$, con un acabado superficial comercial 2B (UNE EN ISO 10088-2). Dicho acabado se obtiene mediante un proceso de laminación en frío, posterior recocido en atmósfera oxidante, decapado y finalmente una pasada de endurecimiento (skin pass).

La composición química y las propiedades mecánicas del acero se indican en las tablas I y II. En la tabla II se establecen también los índices de anisotropía normal, r, y de endurecimiento, n, correspondientes al acero. La rugosidad aritmética media, $\mathrm{Ra}$, medida en el acero es $0,08 \mu \mathrm{m}$.

Tabla I. Composición química (\%) del acero AISI 304 DDQ

Table I. Chemical composition (\%) of AISI 304 DDQ steel

\begin{tabular}{ccccccccc}
\hline $\mathbf{C}$ & $\mathbf{S}$ & $\mathbf{S i}$ & $\mathbf{M n}$ & $\mathbf{C r}$ & $\mathbf{N i}$ & $\mathbf{M o}$ & $\mathbf{V}$ & $\mathbf{C u}$ \\
\hline 0,040 & 0,003 & 0,19 & 1,11 & 17,67 & 9,01 & 0,28 & 0,08 & 0,26 \\
\hline
\end{tabular}

Tabla II. Propiedades mecánicas e índices de conformabilidad del acero AISI 304 DDQ

Table II. Mechanical properties and formability indices of AISI $304 D D Q$ steel

\begin{tabular}{lccccc}
\hline Acero & $\begin{array}{c}\mathbf{R p}_{\mathbf{0}, 2} \\
(\mathbf{M P a})\end{array}$ & $\begin{array}{c}\mathbf{R m} \\
(\mathbf{M P a})\end{array}$ & HV 0,5 & $\mathbf{r}$ & $\mathbf{n}$ \\
\hline 304 DDQ (2B) & 252 & 582 & 174 & 0,906 & 0,244 \\
\hline
\end{tabular}




\subsection{Ensayos de doblado bajo tensión en condiciones de deformación por cortadura pura}

El objeto de este ensayo es simular el comportamiento de la chapa en la zona de doblado en el radio de entrada a la matriz en los procesos de embutición profunda de chapa ${ }^{[11 \text { y } 24]}$. La chapa es sometida a una deformación bajo condiciones de tracción-compresión biaxial, típicas de la acción existente bajo el prensachapas, y simultáneamente es sometida al proceso de doblado. De este modo, la chapa es doblada en condiciones de deformación por cortadura pura o "pure shear" en las que la deformación se produce en el plano principal de la chapa, sin variación en el espesor de la misma. En la figura 1 se indica el esquema de definición del ensayo. La realización del ensayo se ha efectuado mediante un dispositivo desarrollado experimentalmente en el que existe un cilindro de doblado y una matriz con forma de cuña para efectuar los procesos de doblado y de deformación en el plano principal de la chapa, respectivamente. Para evitar el pandeo de la chapa durante la deformación, se aplica una determinada fuerza de cierre, $\mathrm{F}_{\mathrm{N}}$, sobre aquélla. La fuerza de cierre, $F_{\mathrm{N}}$, el ángulo de doblado, $\alpha$, y el radio del cilindro de doblado, $R_{C}$, pueden establecerse como variables de ensayo. La matriz de deformación en forma de cuña, el prensachapas para la aplicación de la fuerza de cierre y el cilindro de doblado se han ejecutado en acero templado F-5250 con una dureza HRC 68. Se han experimentado tres ángulos de doblado, $150^{\circ}, 120^{\circ}$ y $90^{\circ}$, y dos radios de doblado, 3,5 $\mathrm{mm}$ y $6,5 \mathrm{~mm}$.

Las probetas ensayadas se obtuvieron cortando bandas de chapa de acero AISI 304DDQ en estado de recepción, variando la anchura inicial, $\mathrm{h}_{0}$, entre 11,5 y $15 \mathrm{~mm}$. Para poder introducir las muestras en la matriz, se mecanizó un estrangulamiento con la forma de ésta. Teniendo en cuenta que la anchura de la matriz en su extremo más estrecho, $h_{1}$, es $10,80 \mathrm{~mm}$, se practicaron diferentes relaciones de deformación en el plano principal de la chapa, en función de la anchura inicial seleccionada. Una vez que la zona más ancha de la banda sale por la matriz, la relación de deformación practicada, $\varepsilon$, se mantiene constante y de valor indicado en la ecuación (1):

$$
\varepsilon=\ln \frac{h_{0}}{h_{1}}
$$

La velocidad de estirado de la chapa y la presión aparente en la matriz se han considerado como constantes de ensayo, fijando los correspondientes valores en $100 \mathrm{~mm} / \mathrm{min}$ y $3,90 \mathrm{MPa}$, respectivamente. Para mantener constante la presión de cierre en la matriz

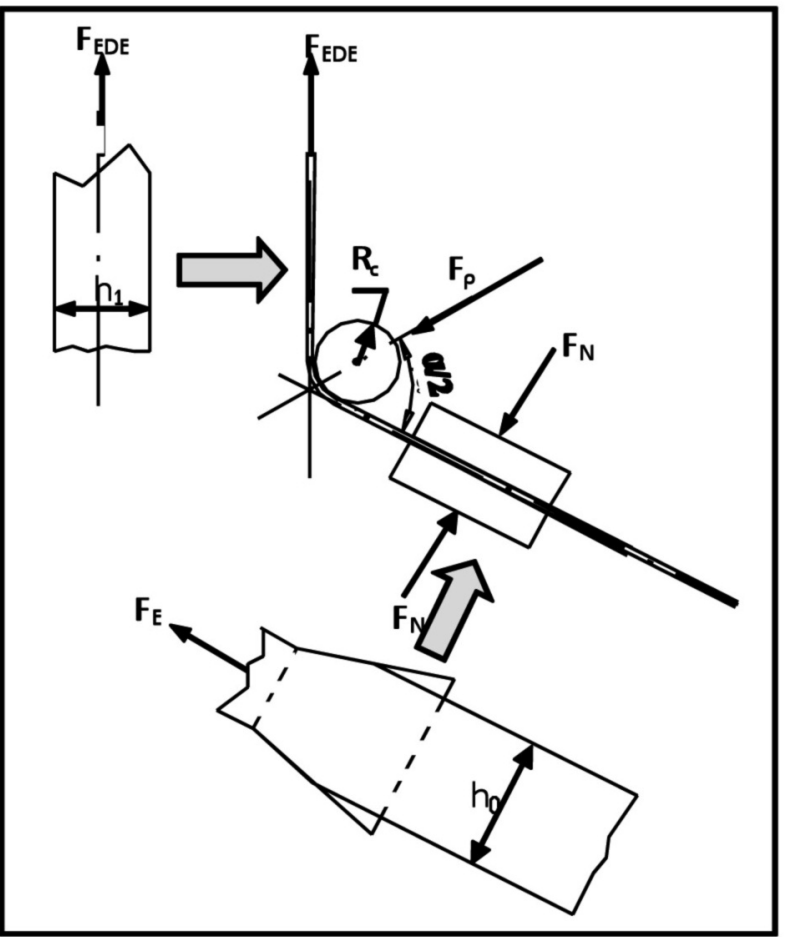

Figura 1. Esquema del ensayo de doblado y estirado[11].

Figure 1. Sketch of bending under tension and pure shear deformation test ${ }^{[11]}$.

se selecciona una diferente fuerza de cierre, $\mathrm{F}_{\mathrm{N}}$, en función de la anchura de la muestra a ensayar, que define una determinada superficie de contacto con la matriz, que es evaluada de manera geométrica.

Como lubricante se ha empleado una mezcla de aceite mineral de baja viscosidad y MoS2 al 50 \% en peso con el fin de reducir el rozamiento existente en las caras laterales de la chapa. Este tipo de lubricante garantiza la existencia de película de lubricante en condiciones de presión elevadas, dando lugar a un coeficiente de rozamiento sensiblemente inferior al que se obtiene mediante la lubricación con aceites minerales ${ }^{[25]}$.

Es posible medir la reacción, $\mathrm{F}_{\mathrm{P}}$, en la bisectriz del ángulo de doblado así como la fuerza precisa para la realización del ensayo, $\mathrm{F}_{\mathrm{EDE}}$. La fuerza involucrada en el proceso de deformación de la banda, $\mathrm{F}_{\mathrm{E}}$, ha sido establecida previamente en función de la deformación practicada $^{[26]}$ y establece la tensión a la entrada del cilindro de doblado o "back stress", $\sigma_{\mathrm{E}}$.

\subsection{Determinación del springback}

Para la determinación de la tendencia al springback del acero tras su conformado, se ha establecido una 
metodología a partir de las muestras ensayadas, (Fig. 2), basada en la geometría de las mismas tras el ensayo de doblado bajo tensión. Las muestras son deformadas a partir del estrangulamiento producido para su inserción en la matriz. Simultáneamente son sometidas a los procesos de doblado, a la entrada del cilindro, y de desdoblado, a la salida del mismo, típicos de este tipo de ensayos. La zona final del material no sufre proceso de doblado alguno debido a la distancia existente desde la salida de la matriz de cuña al cilindro de doblado. Por tanto, se pueden visualizar dos zonas claramente diferenciadas en las muestras ensayadas; existe una zona de mayor longitud que corresponde al material deformado y que ha sufrido el proceso completo de doblado y de desdoblado en el ensayo. La zona más corta, que coincide con el final de la muestra, corresponde al material deformado que no ha sufrido doblado, esto es, que ha quedado justo a la entrada del cilindro de doblado. Lógicamente, la primera parte se correspondería con la zona de pared de un material embutido; la última parte lo hace con la parte del flanco que todavía no ha entrado en la cavidad de la matriz.

La caracterización geométrica de ambas zonas se ha hecho trazando el perfil interior de las muestras ensayadas y determinando mediante la aplicación AUTOCAD ${ }^{\circledR}$ el radio de curvatura en la zona de doblado, $R_{d}$, así como en la de doblado y desdoblado, $R_{p}$, tal como se indica en la figura 3. El método de medición se contrastó con valores medidos en un proyector de perfiles Nikon V12, comprobando que los valores obtenidos eran similares. De este modo se puede establecer un valor de incertidumbre al método del mismo orden de magnitud que el del proyector, esto es, $20 \mu \mathrm{m}$. La ventaja del método indicado es que permite registrar gráficamente la forma final de la chapa y realizar comparaciones visuales.

Se puede establecer la evaluación del springback a partir de los radios medidos, tal y como se deduce en la figura 4. Para la determinación del ángulo que representa la recuperación elástica en la zona de doblado, $\Delta \theta_{1}$, se ha establecido la hipótesis de considerar el arco de contacto chapa-cilindro en la posición de carga como referencia. De este modo, la variación del ángulo cuando cesa la carga o springback se evalúa conforme a la ecuación (2), en la que $\theta_{1 \mathrm{i}}$ y $\theta_{1 \mathrm{f}}$ corresponden a los valores del ángulo de doblado durante el proceso y tras cesar la carga, respectivamente.

$$
\Delta \theta_{1}=\theta_{1 f}-\theta_{1 i}=\theta_{1 i}\left(\frac{\mathrm{R}_{\mathrm{c}}}{\mathrm{R}_{\mathrm{d}}}-1\right)
$$

La zona del material que ha sufrido el proceso completo doblado-desdoblado experimenta una curvatura como consecuencia de la recuperación elástica. Esta curvatura es definida mediante el radio $\mathrm{R}_{\mathrm{p}}$ de forma geométrica independiente de la longitud del material que ha sufrido el proceso ya que dicho valor
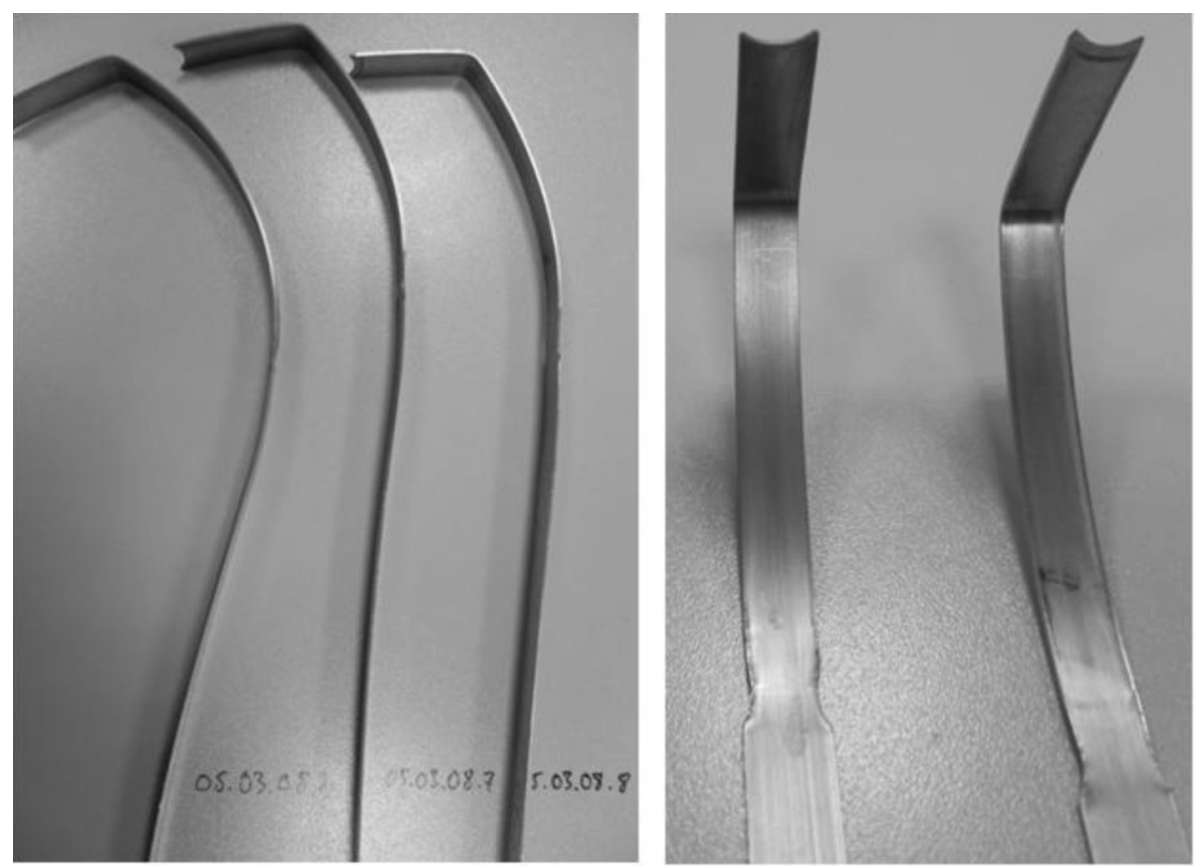

Figura 2. Muestras ensayadas: vista de perfil y vista frontal.

Figure 2. Samples tested: profile view and front view. 


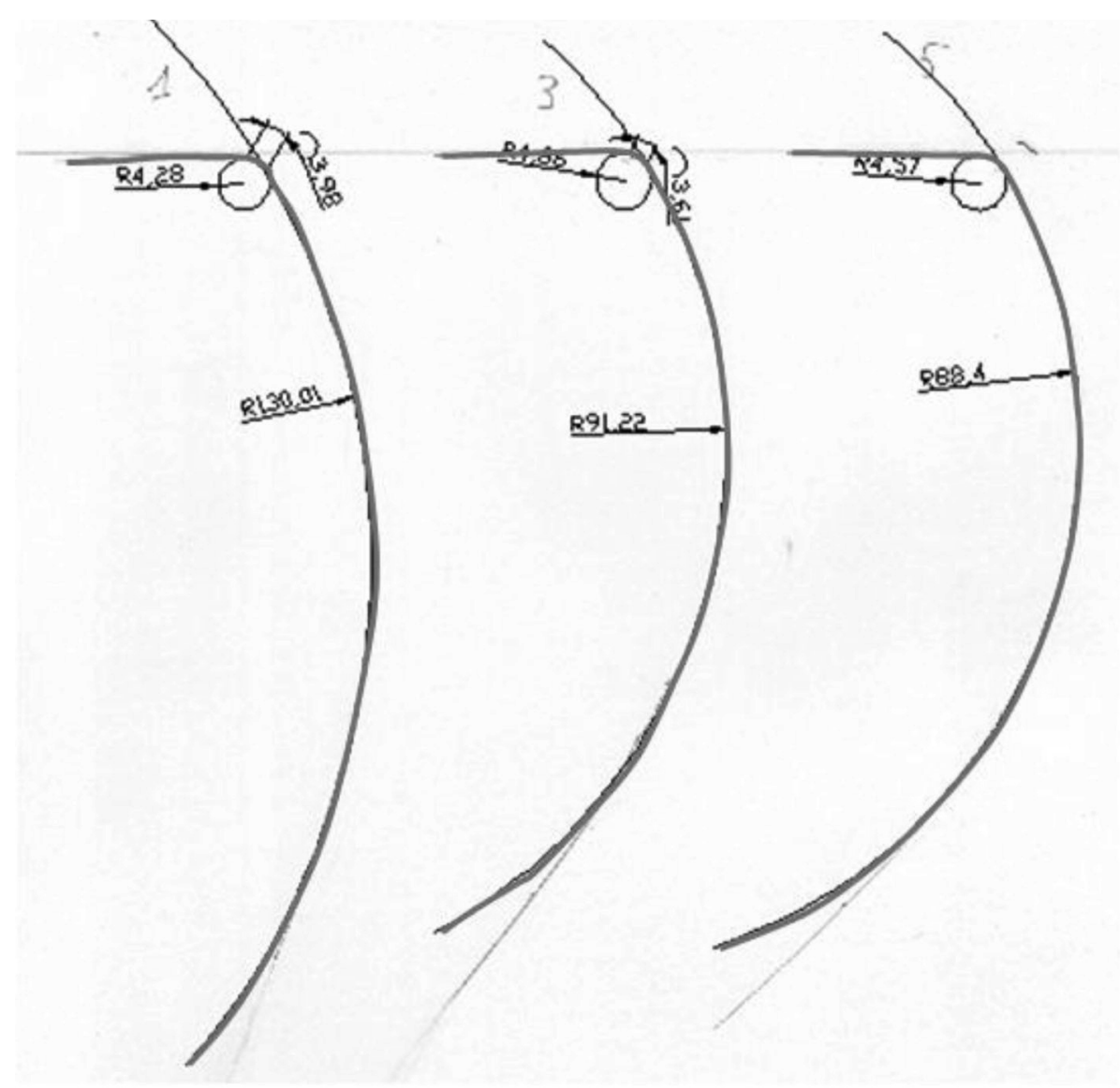

Figura 3. Esquema de la recuperación elástica o springback de dos muestras ensayadas a doblado bajo tensión. Ángulo de doblado $120^{\circ}$ y radio de doblado $3,5 \mathrm{~mm}$.

Figure 3. Elastic recovery or springback corresponding to different samples. Bending angle $120^{\circ}$ and bending radius $3.5 \mathrm{~mm}$.

debe definirse en las inmediaciones del cilindro de doblado. Efectivamente, la longitud de esta zona debe acotarse en correspondencia con la altura de embutición, $\mathrm{H}$, de una copa conformada. El ángulo que representa directamente la recuperación elástica en esta zona, $\theta_{2}$, se establece fijando la longitud de arco correspondiente a la altura de embutición considerada (Ec. (3)):

$$
\Delta \theta_{2}=\frac{\mathrm{H}}{R_{p}}
$$

La relación $R_{c} / R_{d}$ se define factor de recuperación del material por analogía con otros autores ${ }^{[17]}$. De acuerdo con las relaciones anteriores se puede afirmar que existe una relación directa entre el factor de recuperación y la variación del ángulo respecto a la geometría del ensayo en la zona de doblado del material. El radio de curvatura, $R_{p}$, es inverso a la variación del ángulo en la zona de salida en la que la chapa ha sufrido el proceso de doblado y de desdoblado. En cualquier caso $R_{p}$ o su inversa, $1 / R_{p}$, es un indicador que permite establecer tendencias de comportamiento en función de las variables experimentales consideradas.

Finalmente, se puede establecer como indicador del springback el ángulo total de recuperación definido conforme a la ecuación (4). Es importante reseñar que los ángulos de recuperación $\Delta \theta_{1}$ y $\Delta \theta_{2}$ tienen direcciones contrarias, lo que significa que tienen signos contrarios y, por tanto, se compensan en términos relativos.

$$
\Delta \theta=\Delta \theta_{1}+\Delta \theta_{2}
$$

\subsection{Proceso de embutición de copas}

Se ha procedido a embutir copas mediante un dispositivo experimental formado por un conjunto guía- 

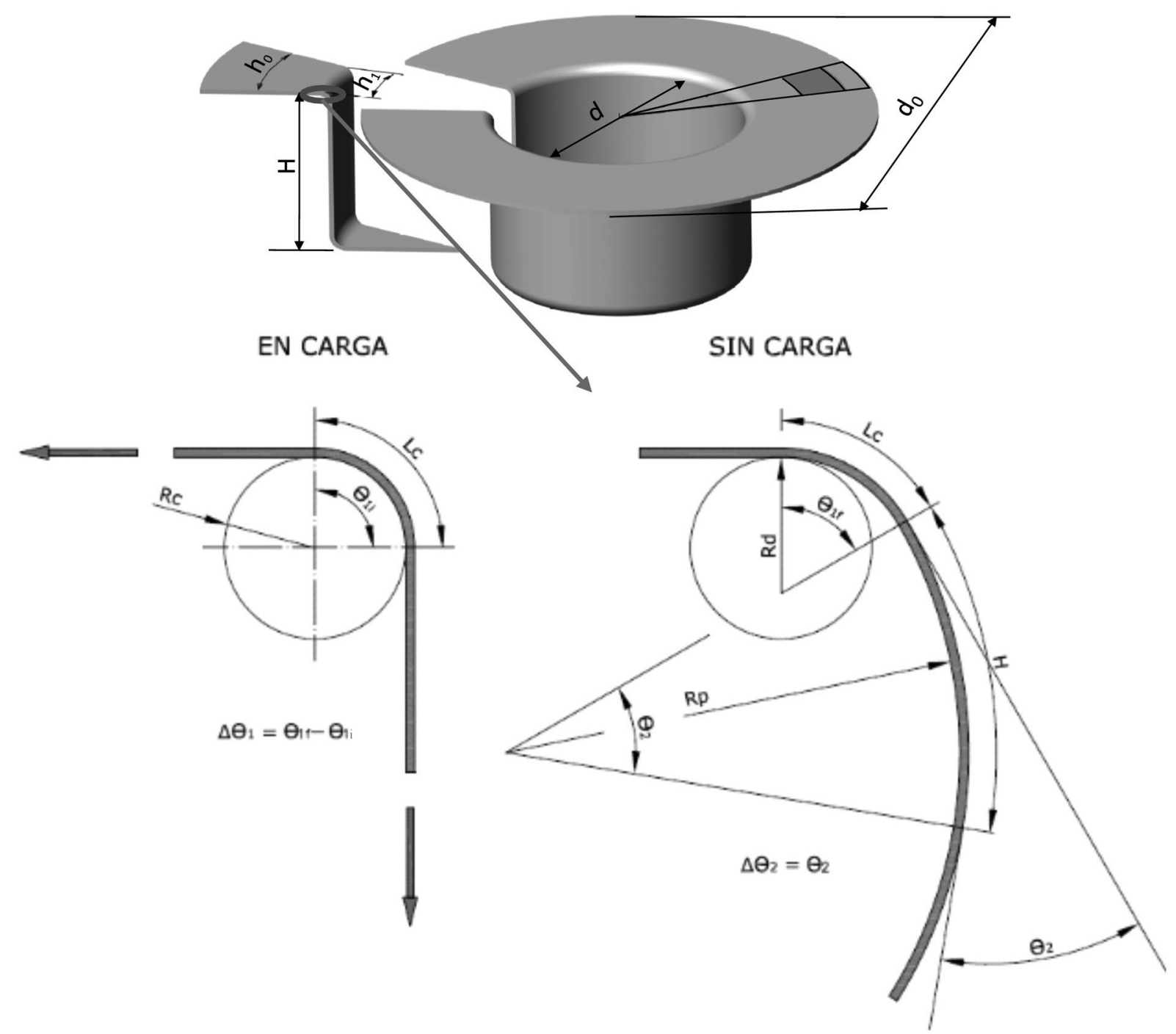

Figura 4. Evaluación geométrica de los ángulos de recuperación elástica conforme a las muestras ensayadas y su correspondencia con la definición de copas embutidas.

Figure 4. Geometric evaluation of the springback angles according to the tested samples and its relationship with the deep-drawn cups.

plataformas para alojar el punzón, el prensachapas y la matriz de embutición. El dispositivo es conectado a un sistema de fuerza hidráulico que es convenientemente controlado. Dicho sistema se emplea para accionar el prensachapas. El dispositivo es alojado en una máquina de tracción universal que actúa como prensa para el accionamiento del punzón. La matriz y el punzón empleados se indican en la tabla III. Dichos elementos son de acero F1552 templado y revenido con una dureza de $68 \mathrm{HRc}$. El radio de acuerdo en la cavidad de la matriz es 2,05 mm.

En la embutición se ha partido de discos de diferente diámetro inicial con el fin de conseguir diferentes relaciones de embutición. La embutición se interrumpió con el fin de conseguir copas con zona de flanco. Lógicamente, la relación de embutición
Tabla III. Dimensiones de la matriz y del punzón empleados para la embutición de copas

Table III. Die and punch dimensions used to axysimmetrical deep-drawing of cups

\begin{tabular}{ccc}
\hline $\begin{array}{c}\text { Diámetro } \\
\text { matriz } \\
\mathbf{D}_{\mathrm{m}}(\mathbf{m m})\end{array}$ & $\begin{array}{c}\text { Diámetro } \\
\text { punzón } \\
\mathbf{D}_{\mathrm{p}}(\mathbf{m m})\end{array}$ & $\begin{array}{c}\text { Juego } \\
(\mathbf{m m})\end{array}$ \\
\hline 59,75 & 58 & 0,87 \\
\hline
\end{tabular}

no se puede calcular en este caso directamente como la razón entre el diámetro inicial del disco de partida, $\mathrm{d}_{0}$, y el diámetro de la copa obtenida, $\mathrm{d}$, sino que se 
obtuvo a partir de la altura de embutición, teniendo en cuenta la geometría de la copa.

La medición de los radios $R_{1}$ y $R_{p}$ se efectuó cortando las copas diametralmente en la dirección de laminación. Se comprobó que los resultados obtenidos eran similares partiendo de media copa o de una banda de $10 \mathrm{~mm}$ de anchura obtenida de la copa (Fig. 5). Las mediciones se efectuaron en un proyector de perfiles Nikon V12, obteniendo los radios mencionados seleccionando tres puntos del perfil interior en las zonas correspondientes. El procesador del proyector da el resultado del radio de la circunferencia que pasa por los puntos marcados.

\section{RESULTADOS Y DISCUSIÓN}

\subsection{Resultados de los ensayos de doblado bajo tensión}

En las figuras 6 y 7 se representan el factor de recuperación, $R_{c} / R_{d}$, y el indicador $1 / R_{p}$, respectivamente, en función de la relación de deformación experimentada por el material previa al proceso de doblado. Como puede observarse, el factor de recuperación y por tanto el ángulo de recuperación elástica en el doblado, $\Delta \theta_{1}$, es independiente de la deformación experimentada por el material. Como aproximación se puede establecer que la razón $h_{0} / h_{1}$ es equivalente a la razón de embutición $\mathrm{d}_{0} / \mathrm{d}$, por lo que se puede afirmar que el factor de recuperación es independiente de la relación de embutición. La invariabilidad del factor de recuperación se puede explicar a partir del efecto contrapuesto del endurecimiento por deformación que experimenta el material y de la tensión

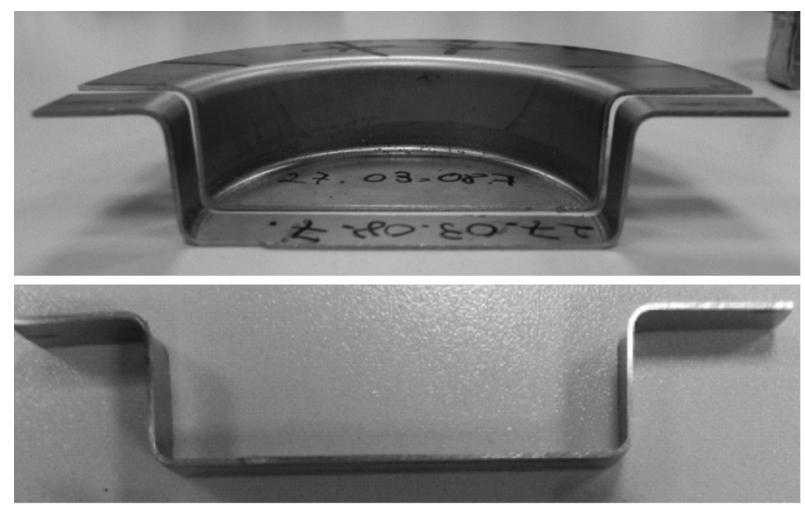

Figura 5. Detalle del corte efectuado para la medición de los radios $R_{1}$ y $R_{\rho}$ en copas embutidas.

Figure 5. Detail of the transversal cut of a deep-drawn cup for measuring $R_{1}$ y $R_{\rho}$. de estirado necesaria para producir la deformación y que determina la tensión presente en el proceso de doblado. La influencia de la relación de deformación se manifiesta en el aumento del límite elástico del material por el endurecimiento por acritud experimentado. Como consecuencia de ello, una mayor relación de deformación debería conducir a una disminución del factor de recuperación o, en consecuencia, a un incremento del springback en dicha zona. Del mismo modo, el aumento de la relación de deformación se manifiesta en un incremento de las tensiones existentes en el proceso de doblado. Como es sabido, un aumento de la tensión de estirado durante el doblado se caracteriza por una disminución de la recuperación elástica del material. Los resultados obtenidos para la variación de $R_{c} / R_{d}$ mostrados en la figura 6 se justifican si se considera que los efectos del endurecimiento del material y las tensiones de estirado debidos a la relación de deformación se compensan.

La curvatura obtenida en la zona de dobladodesdoblado del material presenta un comportamiento similar para ángulos de doblado superiores a $90^{\circ}$, esto es, ángulos de doblado más suaves, pudiendo inferirse, además, un equilibrio entre el nivel de tensión implicado en el estirado y el aumento del límite elástico o endurecimiento experimentado por el material, tal como se ha indicado anteriormente. La curvatura observada para un ángulo de doblado de $90^{\circ}$ es decreciente con la deformación. El fenómeno de desdoblado se produce a la salida del cilindro. El material correspondiente a dicha zona está sometido al rozamiento del mismo en el cilindro y las tensiones que soporta el material localmente son mayores ${ }^{[4]}$. Este efecto es considerablemente mayor en el doblado a $90^{\circ}$ que con ángulos menos exigentes, en los que el contacto chapa-cilindro es considerablemente menor. La fuerza de contacto también es considerablemente mayor en $90^{\circ}$ que en $120^{\circ}$ y $150^{\circ}[11$ y 24]. Dado que el grado de deformación es independiente del ángulo de doblado, se deduce que las tensiones de estirado existentes para cada uno de los ángulos ensayados influyen significativamente en la recuperación elástica experimentada por el material a la salida del cilindro de doblado. El nivel de tensiones involucradas en $120^{\circ}$ y $150^{\circ}$ es similar ${ }^{[24]}$, por lo que el efecto combinado de éstas y del endurecimiento del material hace que la recuperación a la salida del cilindro sea independiente de la relación de deformación y de valor similar en ambos casos. Los valores de recuperación para $90^{\circ}$ son considerablemente menores, lo que es consistente con un nivel mayor de tensiones para este último ángulo de doblado. Del mismo modo, conforme aumenta la relación de deformación parece existir cierta preponderancia del efecto de la tensión sobre el endurecimiento del material. 
Así mismo, en la figura 6 se observa que no existe una influencia significativa del radio de doblado sobre el factor de recuperación. Efectivamente, los valores obtenidos para un ángulo de doblado de $90^{\circ}$ son similares para los dos radios experimentados, 6,5 y $3,5 \mathrm{~mm}$. Para un ángulo de doblado de $120^{\circ}$ tampoco se observa una diferencia significativa, si bien es algo mayor. La tendencia observada en este último caso es también esperable ya que el factor de recuperación aumenta para el radio de doblado menor. A la vista de los resultados, parece que un radio de $6,5 \mathrm{~mm}$ presenta valores de recuperación lo suficientemente elevados como para justificar que no se produzca una influencia notable si se disminuye dicho radio.

Los resultados experimentales obtenidos se han comparado con los resultados analíticos determinados mediante la ecuación (5) desarrollada por Gardiner ${ }^{[3]}$, que determina la recuperación elástica considerando las propiedades que definen el comportamiento elástico de los materiales utilizados. Según esto, el factor de recuperación, $R_{c} / R_{d}$, depende de la tensión de fluencia, $\mathrm{R}_{\mathrm{p}}$, del módulo elástico del material, E, y del espesor de la chapa, t.

$$
\frac{R_{c}}{R_{d}}=4 \cdot\left(\frac{R_{c} R_{p}}{t E}\right)^{3}-3 \cdot\left(\frac{R_{c} R_{p}}{t E}\right)+1
$$

La ecuación de Gardiner, obtenida para procesos de doblado al aire, aplicada al acero experimentado, al espesor de chapa ensayado y para un radio de doblado de 6,5 $\mathrm{mm}$ conduce a un valor de 0,967 para el factor de recuperación. Los resultados obtenidos para $90^{\circ}$ son bastante próximos a dicho valor, aun considerando que las condiciones de ensayo son muy diferentes en lo relativo al estado de tensiones al que se somete al material. En definitiva, esto demuestra que los valores de recuperación para un radio de doblado de 6,5 mm están próximos al techo de recuperación previsible en estas condiciones.

En relación a la curvatura experimentada a la salida del cilindro, se observa una influencia algo más significativa del radio de doblado para ángulos superiores a $90^{\circ}$. Así, para $120^{\circ}$ y radio $3,5 \mathrm{~mm}$ se observa incluso, la misma tendencia a disminuir la recuperación con la deformación que la indicada para un ángulo de $90^{\circ}$, lo que es coherente con la justificación establecida con anterioridad en esta zona del ensayo.

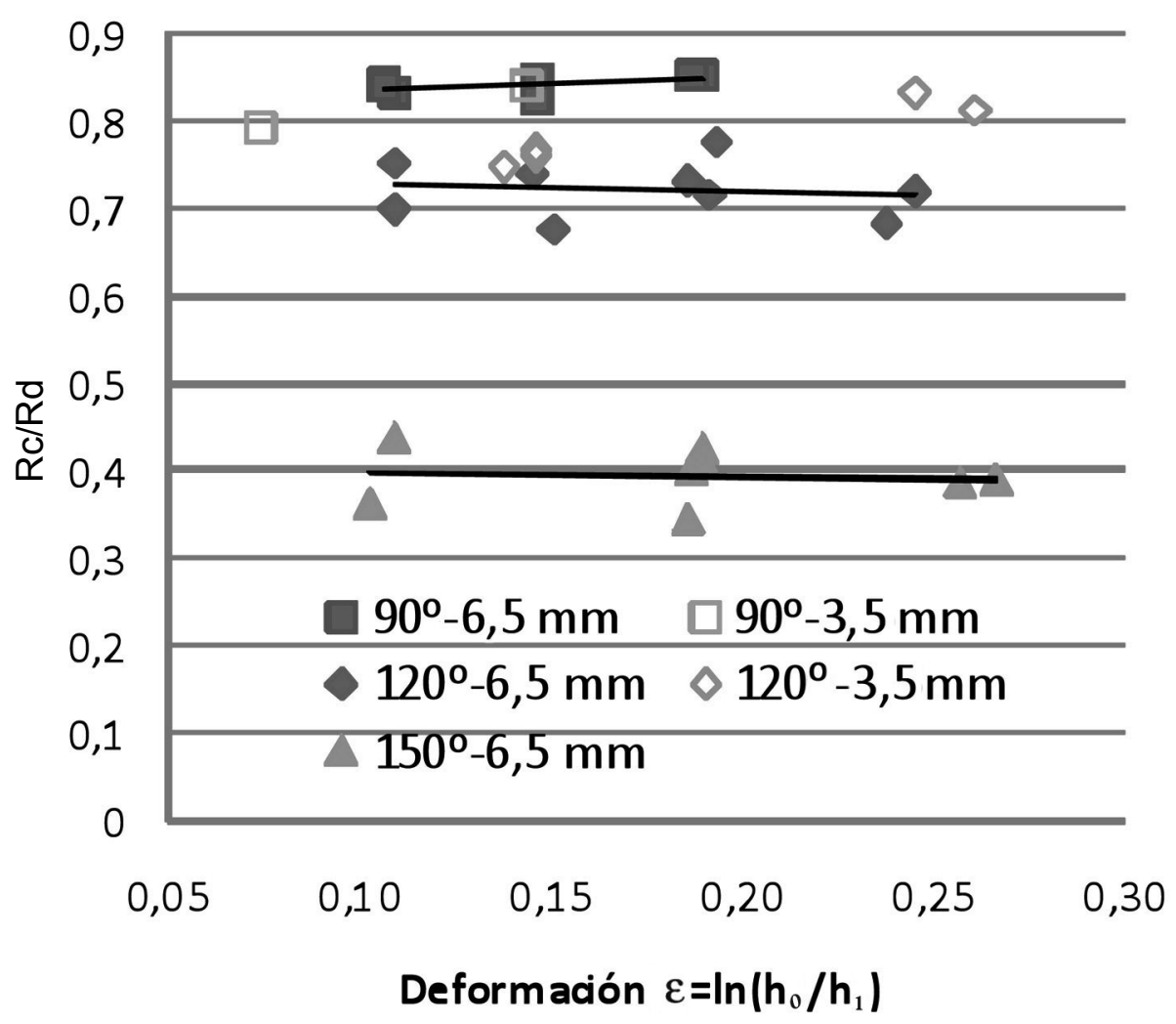

Figura 6. Valor del factor de recuperación $R_{c} / R_{d}$ en función de la relación de deformación que experimenta el material durante el ensayo.

Figure 6. Recovery factor $R_{c} / R_{d}$ value as a function of the strain ratio experimented by the material during the test. 


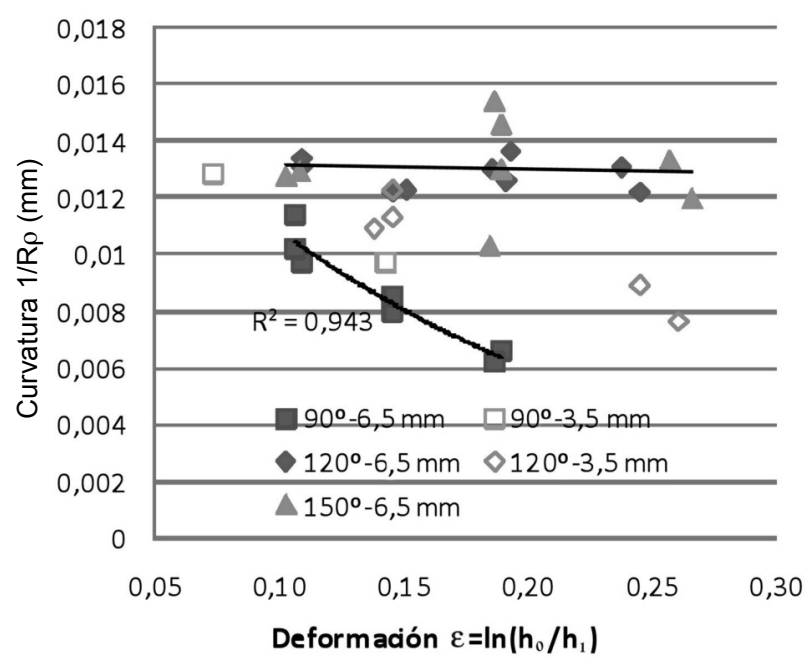

Figura 7. Valor del radio de curvatura $R_{\rho}$ en función de la relación de deformación que experimenta el material durante el ensayo.

Figure 7. Curvature radius $R_{\rho}$ value as a function of the strain ratio experimented by the material during the test.

\subsection{Exportación de los resultados al proceso de embutición de copas}

La analogía geométrica del factor de recuperación con la zona de unión de las zonas de pared y del flanco resulta evidente, así como la identificación del ángulo $\theta_{1 \mathrm{f}}$ y la evaluación de la recuperación en dicha zona $\Delta \theta_{1}$ conforme a la ecuación (2). En la figura 8 se representa el valor $\Delta \theta_{1}$ en función de la deformación realizada para los diferentes ángulos y radios de doblado experimentados.

La evaluación del ángulo $\Delta \theta_{2}$ precisa la selección de una longitud de arco que esté definida a partir de la geometría de una copa para la relación de embutición establecida. Por tanto, es preciso exportar el ensayo definido sobre bandas de chapa a la deformación en embutición de copas. Así, considerando la equivalencia de áreas correspondientes al plano principal de la chapa para una copa embutida de diámetro d, a partir de un disco de chapa de diámetro $_{0}$, (Fig. 4), se obtiene la conocida ecuación (6) que relaciona geométricamente las diferentes dimensiones de la copa y del diámetro del disco inicial.

$$
\mathrm{d}_{0}^{2}=\mathrm{d}^{2}+4 \mathrm{dH}_{\mathrm{d}}
$$

Estableciendo que la relación de embutición $\mathrm{d}_{0} / \mathrm{d}$ es equivalente a la relación $h_{0} / h_{1}$ y sustituyendo en la ecuación (6) dicha igualdad, se obtiene la ecuación

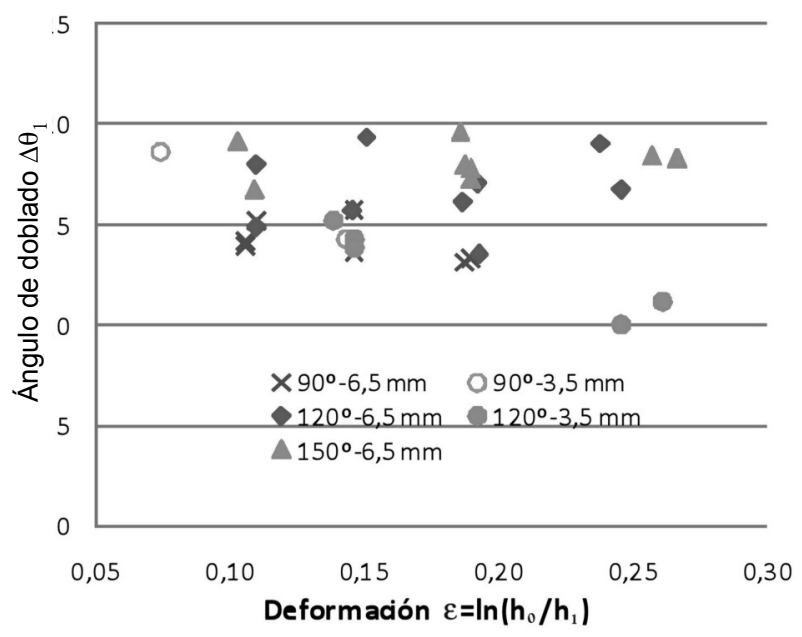

Figura 8. Variación del ángulo de doblado $\Delta \theta_{1}$ en función de la deformación.

Figure 8. Bending angle variation $\Delta \theta_{1}$ as a function of the deformation-value.

(7) que expresa la altura de embutición $\mathrm{H}_{\mathrm{d}}$ en función de la relación de embutición $\mathrm{h}_{\mathrm{o}} / \mathrm{h}_{1}$. $\mathrm{H}_{\mathrm{d}}$ puede también ser expresado en función del diámetro de la copa y de una constante, $K_{\varepsilon}$, dependiente de la deformación del material.

$$
H_{d}=\left(\frac{h_{0}^{2}}{h_{1}^{2}}-1\right) \frac{d}{4}=\frac{1}{4 e^{2 \varepsilon} h} d=K_{\varepsilon} \cdot d
$$

No obstante, el arco comienza a partir del radio de curvatura definido mediante el radio de doblado $\mathrm{R}_{\mathrm{c}}$ (Fig. 4), por lo que la longitud del arco $\mathrm{H}$ a considerar para la obtención del ángulo de recuperación elástica $\Delta \theta_{2}$ se puede hacer de manera aproximada restando a $\mathrm{H}_{\mathrm{d}}$ el valor del radio $\mathrm{R}_{\mathrm{c}}$, conforme se establece en la ecuación (8):

$$
\mathrm{H}=\mathrm{H}_{\mathrm{d}}-\mathrm{R}_{\mathrm{c}}
$$

El ángulo de recuperación elástica $\Delta \theta_{2}$ correspondiente a una determinada altura $\mathrm{H}$ podría expresarse de acuerdo a la ecuación (9):

$$
\Delta \theta_{2}=\frac{K_{\varepsilon}}{R_{p}} \cdot d
$$

En la figura 9 se representan los valores para $\Delta \theta_{2}$ correspondientes a la exportación de los resultados obtenidos para $1 / R_{p}$ a operaciones de estampación 

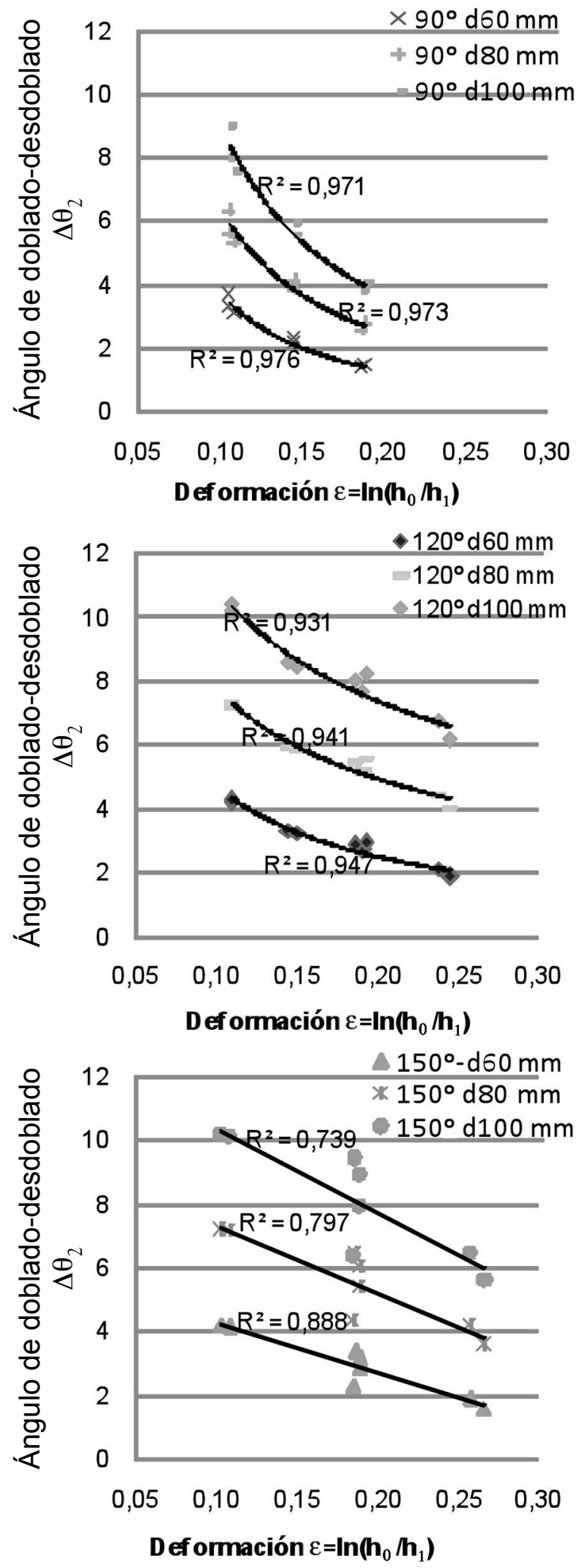

Figura 9. Simulación del valor de recuperación $\Delta \theta_{2}$ a partir de valores de referencia $\mathrm{H}$ correspondientes a copas de diferentes diámetros conforme a los resultados obtenidos en el test de doblado bajo tensión.

Figure 9. $\Delta \theta_{2}$ values that are been obtained for arcs lengths $\mathrm{H}$ corresponding to cups of different diameters according to the experimental results of bending under tension tests. de copas con radio de entrada a la matriz de $6,5 \mathrm{~mm}$ y con ángulos de doblado de $150^{\circ}, 120^{\circ}$ y $90^{\circ}$. Se han simulado diferentes diámetros de copa. Lógicamente, $\Delta \theta_{2}$ es mayor para diámetros de copas mayores ya que al mantener constante la relación de embutición, éstas presentan mayores alturas y el arco $\mathrm{H}$, también mayor, conduce a mayores valores de recuperación.

Los valores de recuperación obtenidos en los ensayos también permiten establecer un springback similar en la zona de pared para ángulos de doblado de $150^{\circ}$ y $120^{\circ}$, siendo algo inferiores para un ángulo de $90^{\circ}$ (Fig. 9). En cualquier caso, la influencia del ángulo de doblado sobre $\Delta \theta_{2}$ resulta despreciable para el radio de doblado indicado.

\subsection{Resultados de embutición de copas}

Se ha medido el factor de recuperación en copas de $60 \mathrm{~mm}$ de diámetro con diferentes relaciones de embutición. Los resultados obtenidos se indican en la figura 10 junto con los correspondientes a los ensayos efectuados a $90^{\circ}$ con el fin de contrastar los valores obtenidos. Como se puede observar, se obtiene una buena correspondencia entre los valores arrojados por los ensayos y los obtenidos en copas embutidas a pesar del menor radio de doblado empleado en la embutición de copas, tal y como era esperable por la influencia menor observada para el

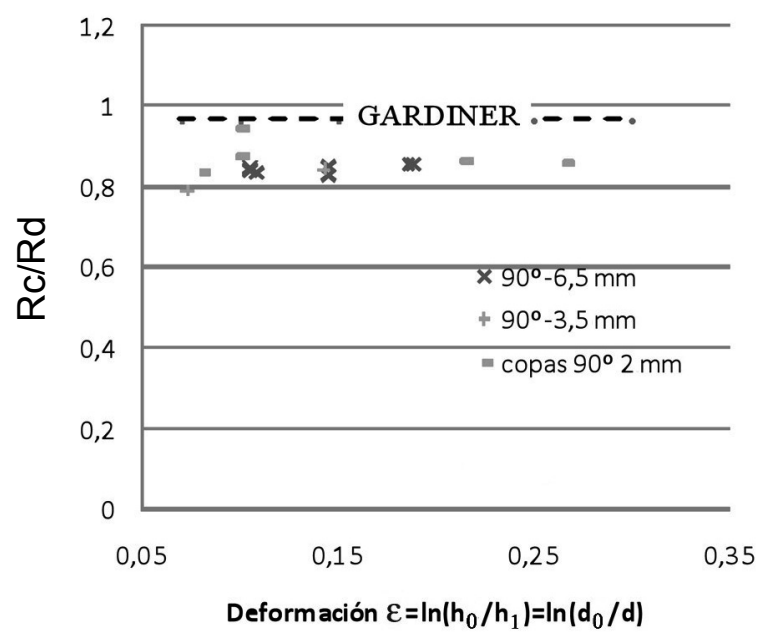

Figura 10. Comparación del factor de recuperación $R_{c} / R_{d}$ medido en copas embutidas y en los ensayos de doblado bajo tensión a $90^{\circ}$.

Figure 10. Comparative results for the recovery index $R_{c} / R_{d}$ measured in deep-drawn cups and with the $90^{\circ}$ bending under tension tests. 
radio de doblado. En la figura 10 también se representa el valor de recuperación correspondiente a la ecuación de Gardiner para un radio de doblado de 6,5 $\mathrm{mm}$.

En relación al indicador $1 / R_{p}$, en la figura 11 se muestran los valores obtenidos en copas embutidas. En la figura 12 se establece en detalle algunas de las secciones medidas. Los valores obtenidos son más bajos que los medidos en los ensayos de doblado bajo tensión. Estas diferencias podrían deberse al hecho de que en la embutición de copas la relación de embutición experimentada por la chapa es diferente según sea la altura de pared considerada. Así, el springback suele ser menor cerca del fondo de la copa ya que el material fue doblado con una relación de embutición local menor ${ }^{[27]}$. El ensayo efectuado a las bandas de chapa realiza una relación de embutición constante e igual a la máxima en toda su dimensión. No obstante, el ensayo permitiría evaluar comparativamente las diferencias de comportamiento de diversos materiales en esta zona. En cualquier caso, los resultados

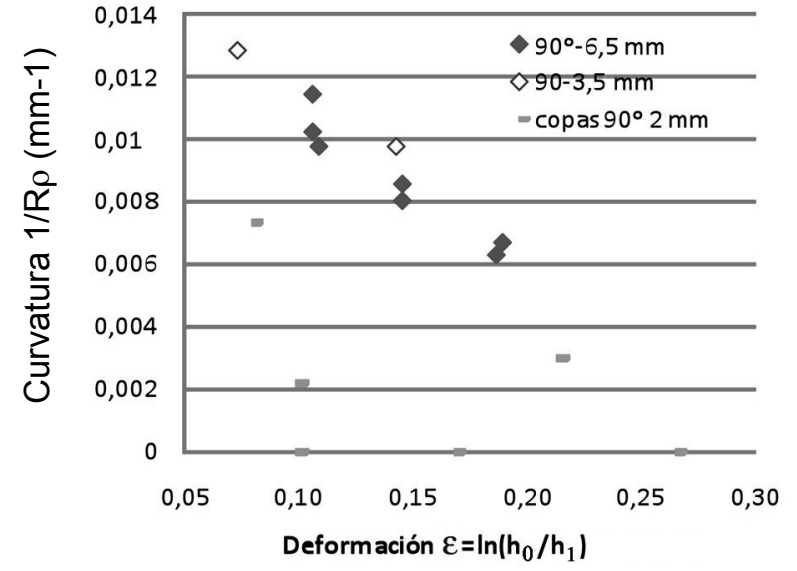

Figura 11. Comparación del valor $1 / R_{\rho}$ medido en copas embutidas y en los ensayos de doblado bajo tensión a $90^{\circ}$.

Figure 11. Comparative results for the parameter $1 / R_{\rho}$ measured in deep-drawn cups and with the $90^{\circ}$ bending under tension tests.
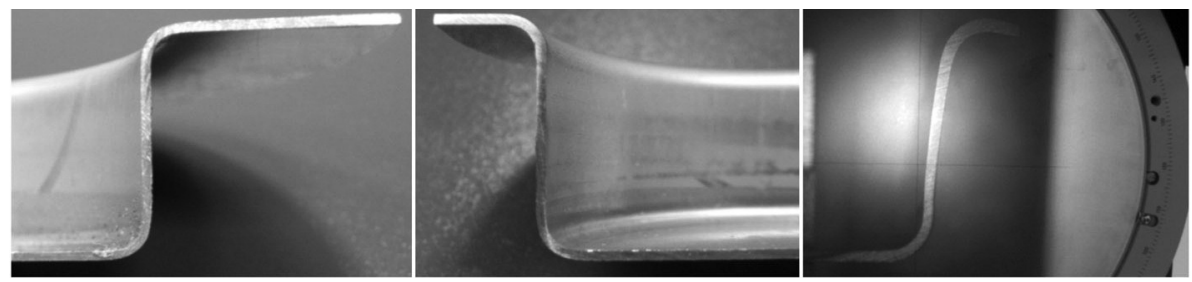

Figura 12. Detalle de algunas de las secciones medidas para la determinación del factor de recuperación y el valor $1 / R_{\rho}$.

Figure 12. Detail of some section used to determine de recovery index and $1 / R_{\rho}$ value.

obtenidos en los ensayos son del mismo orden de magnitud que los obtenidos por otros autores en operaciones de stretch-bending ${ }^{[28}$ y 29$]$.

\section{CONCLUSIONES}

- En el presente trabajo se ha desarrollado una metodología para la evaluación del springback de metales sometidos a un estado multiaxial típico de los procesos de embutición de chapa.

- El ensayo propuesto permite evaluar por separado las condiciones de recuperación en el doblado y en la zona de salida del ensayo correspondientes a la zona del ala y de la pared, respectivamente, en un elemento embutido. Para ello, se ha establecido el factor de recuperación $\mathrm{Rc} / \mathrm{Rd}$ y el valor $1 / R_{\rho}$ que definen ambas zonas.
- El ensayo permite evaluar la influencia del ángulo de doblado, del radio de doblado y de la relación de embutición bajo condiciones de deformación típicas de cortadura pura o "pure shear".

- Los resultados obtenidos permiten establecer que no existe influencia de la relación de embutición en los márgenes considerados. La influencia del radio de doblado, para los valores ensayados, también es menor.

- Los resultados obtenidos para el factor de recuperación en la zona de doblado reproducen bastante bien los obtenidos en copas embutidas. Como era previsible, la zona de doblado y desdoblado presenta una recuperación menor en copas embutidas que en los ensayos. No obstante, el ensayo permite determinar tendencias de comportamiento en dicha zona, en la que la recuperación elástica del material suele ser menor. 


\section{REFERENCIAS}

[1] W. Gang y R.H. Wagoner, Int. J. Mech. Sci. 46 (2004) 1.097-1.113.

[2] X.A. Yang y F. Ruan, Int. J. Mech. Sci. 53 (2011) 399-406.

[3] F.J. Gardiner, Trans. ASME (1958) 79.

[4] Z. Marciniak, J.L. Duncan y S.J. Hu, Mechanics of Sheet Metal Forming, Ed. ButterworthHeinemann, $2^{\text {nd }} E d$, UK, 2002, pp. 92-94.

[5] F.J. Avellaneda, V. Miguel-Eguía, J. Coello, A. Martínez y A. Calatayud, Adv. Mat. Res. 498 (2012) 13-18.

[6] Z. Tekiner, J. Mater. Process. Tech. 145 (2004) 109-117

[7] D. Zhang, Z. Cui, X. Ruan y Y. Li, Comp. Mater. Sci. 38 (2007) 707-715.

[8] D. Leu, J. Mater. Process. Tech. 66 (1997) 9-17.

[9] Ö. Tekaslan, U. Seker y A. Özdemir, Mat. \& Des. 27 (2006) 251-258.

[10] M.L. García-Romeu, J. Ciurana y I. Ferrer, J. Mater. Process. Tech. 191 (2007) 174-177.

[11] V. Miguel, J. Coello, A. Calatayud, M.C. Manjabacas, A. Martínez y C. Ferrer, J. Mater. Process. Tech. 209 (2009) 1.588-1.596.

[12] I. Ragai, D. Lazim y J.A. Nemes, J. Mater. Process. Tech. 166 (2005) 116-127.

[13] F. Pourboghrat y E. Chu, J. Mater. Process. Tech. 50 (1995) 361-374.

[14] Y.E. Ling, H.P. Lee y B.T. Cheok, J. Mater. Process. Tech. 168 (2005) 296-302.

[15] S.K. Panthi, N. Ramakrishnan, K.K. Pathak y J.S. Chouhan, J. Mater. Process. Tech. 186 (2007) 120-124.
[16] V. Esat, H. Darendeliler y M.I. Gokler, Mat. \& Des. 23 (2002) 223-229.

[17] W.D. Carden, L.M. Geng, D.K. Matlock y R.H. Wagoner, Int. J. Mech. Sci. 44 (2002) 79-101.

[18] J.F. Wang, R.H. Wagoner, W.D. Carden, D.K. Matlock y F. Barlat, Int. J. Plasticity 20 (2004) 2.209-2.232.

[19] J.F. Wang, R.H. Wagoner, D.K. Matlock y F. Barlat, Int. J. Solids. Struct. 42 (2005) 1.2871.307.

[20] T.B. Hilditch, J.G. Speer y D.K. Matlock, J. Mater. Process. Tech. 182 (2007) 84-94.

[21] K. Hanaki y K. Kato, Adv. Tech. Plasticity 1 (1984) 581-586.

[22] G.J. Coubrough, M.J. Alinger y C.J. Van Tyne, J. Mater. Process. Tech. 130-131 (2002) 69-75.

[23] EL-Megharbel, A. EL-Domiaty y M. Shaker, J. Mater. Process.Tech. 24 (1990) 191-200.

[24] J. Coello, V. Miguel, C. Ferrer, A. Calatayud y A. Martínez, Rev. Metal. 48 (2012) 264 . 276.

[25] J. Coello, V. Miguel, C. Ferrer, A. Calatayud y A. Martínez, Rev. Metal. 44 (2008) 503-512.

[26] J. Coello, V. Miguel, A. Calatayud, A. Martínez y C. Ferrer, Rev. Metal. 46 (2010) 435-445.

[27] W.F. Hosford y R.M. Caddell, Metals Forming. Mechanical and Metallurgy, $3^{\text {rd }}$ Ed., Cambridge University Press, UK, 2007, p. 231.

[28] Y.H. Moon, D.W. Kim y C.J. Van Tyne, Int. J. Mech. Sci. 50 (2008) 666-675.

[29] R. Hino, Y. Goto y F. Yoshida, J. Mater. Process. Tech. 139 (2003) 341-347. 